\title{
Recomendações do enunciado CONSORT para o relato de estudos clínicos controlados e randomizados
}

\author{
The CONSORT statement instructions for \\ reporting randomized clinical trials
}

\author{
Jaqueline Martins ${ }^{1}$, Letícia M. Sousa ${ }^{1}$, Anamaria S. Oliveira ${ }^{2}$
}

\begin{abstract}
RESUMO
O raciocínio clínico e a tomada de decisão pelo profissional de saúde devem ter como base as evidências científicas relevantes e não apenas a intuição ou experiências clínicas prévias não-sistematizadas. Estas evidências são adequadamente obtidas a partir de estudos clínicos controlados randomizados (ECRs), cujas informações são frequentemente relatadas de forma incompleta, prejudicando a identificação de possíveis erros metodológicos. Com intuito de aprimorar o relato de ECR, um grupo de cientistas e editores de periódicos médicos elaborou o enunciado CONSORT, constituído de uma lista de checagem com 22 itens e um fluxograma dos participantes. Atualmente, o CONSORT se encontra traduzido em diversas línguas e em versões adaptadas para atender aos diferentes modelos de estudos. O presente trabalho pretendeu, portanto, facilitar a compreensão de tais instruções, assim como sua divulgação na língua portuguesa, ao abordar brevemente os principais aspectos contidos na versão revisada do enunciado CONSORT.
\end{abstract}

Palavras-chave: CONSORT. Ensaios Clínicos Controlados como Assunto. Ensaio Clínico Controlado Aleatório. Ensaio Controlado Aleatório. Medicina Baseada em Evidências.

Informações mais detalhadas sobre os conceitos apresentados e discutidos brevemente no presente trabalho podem ser encontradas em Altman et al. 2008 e Boutron et al. 2008. Acesse também o endereço eletrônico: http://www.consortstatement.org/

\section{Introdução}

Atualmente, as práticas em saúde são norteadas pelo conceito da medicina baseada em evidências, a qual preconiza que o raciocínio clínico e a tomada de decisão pelo profissional de saúde sejam fundamentados em evidências científicas relevantes e não apenas com base na intuição ou em experiências clínicas prévias não-sistematizadas. ${ }^{1,2,3}$ Sob esta perspectiva, o profissional de saúde deve estar apto a identificar, na literatura disponível, estudos que forneçam informações válidas e confiáveis relativas ao problema em questão. A avaliação crítica desses estudos, entretanto, não depende somente da habilidade do profissional em interpretar seus resultados, mas também da ma-
1. Mestranda. Departamento de Biomecânica, Medicina e Reabilitação do Aparelho Locomotor. Faculdade de Medicina de Ribeirão Preto - USP. Laboratório de Análise da Postura e do Movimento Humano - LAPOMH

2. Docente. Departamento de Biomecânica, Medicina e Reabilitação do Aparelho Locomotor. Faculdade de Medicina de Ribeirão Preto - USP. Laboratório de Análise da Postura e do Movimento Humano - LAPOMH
Correspondência: Profa. Dra. Anamaria Siriani de Oliveira Departamento de Biomecânica, Medicina e Reabilitação do Aparelho Locomotor da FMRP-USP. Avenida dos Bandeirantes, 3900. 14049-900 - Ribeirão Preto / SP

Artigo recebido em 18/12/2008 Aprovado em 20/05/2009 
neira como as informações são descritas pelos pesquisadores e responsáveis por sua divulgação. O relato incompleto ou inadequado de informações sobre o planejamento e condução do estudo prejudica a identificação de possíveis erros metodológicos, assim como, dificulta o uso de suas conclusões pelos interessados, uma vez que estes não conseguem avaliar criticamente sua aplicabilidade clínica. ${ }^{4,5}$

A preocupação com o conteúdo das publicações científicas é tema de discussão desde o final dos anos 70, quando foi primeiramente abordada pelo Comitê Internacional de Editores de Periódicos Médicos (International Committee of Medical Journal Editors - ICMJE) por meio da divulgação de recomendações que deveriam ser consideradas ao se submeter um trabalho para apreciação em periódicos médicos. ${ }^{6}$ Algumas iniciativas semelhantes foram desenvolvidas nas duas últimas décadas, publicadas em forma de tutoriais ou guias com instruções aos autores sobre o relato transparente de estudos clínicos controlados randomizados (ECR), estudos sobre acurácia diagnóstica, assim como de revisões sistemáticas e meta-análises de ECR e de estudos observacionais, entre outros. ${ }^{5,7-10}$ Apesar destes esforços, o relato inadequado ainda é comumente encontrado em todas as áreas de pesquisa em saúde, prejudicando assim a qualidade e utilidade das informações divulgadas na literatura. ${ }^{7}$

Falhas frequentes no relato de ECRs, considerados padrão-ouro para fornecer evidências sobre a eficácia relativa às intervenções terapêuticas, estimularam um grupo de editores e pesquisadores a formularem o enunciado CONSORT (Consolidated Standards of Reporting Trials). ${ }^{4,11}$ Publicado em 1996 e revisado em 2001, este conjunto de critérios auxilia os autores a aperfeiçoar a descrição de seus achados através do emprego de uma lista de checagem de 22 itens e um diagrama representando o fluxo dos participantes durante cada estágio do estudo. ${ }^{4} \mathrm{O}$ enunciado CONSORT facilita a interpretação crítica dos resultados, pois, permite que o leitor conheça detalhes sobre o desenho do estudo, seu modo de condução e o tipo de análise utilizada. Adicionalmente, evita a omissão de possíveis erros sistemáticos que comprometeriam a validade e confiabilidade dos resultados e consequentemente, sua aplicabilidade dentro do contexto da medicina baseada em evidências.

Avaliações associando a adoção do enunciado CONSORT com a melhora da qualidade do relato de $\operatorname{artigos~publicados~}^{12,13}$ têm contribuído progressiva- mente para seu maior endossamento por jornais médicos, que atualmente representam mais de 300 , e por grupos editoriais como Council of Science Editors, ICMJE e World Association of Medical Editors (WAME). Neste sentido, a fim de reforçar sua utilização, o enunciado CONSORT foi traduzido para diferentes línguas, alcançando o número de dez traduções, e apresenta várias extensões que abordam de forma mais apropriada ECR caracterizados por diferentes desenhos, dados e intervenções. Atualmente, as extensões do enunciado CONSORT se direcionam a estudos clínicos desenhados em aglomerado (Cluster trials), estudos de equivalência e não-inferioridade, estudos de intervenções com ervas medicinais, intervenções não-farmacológicas, referentes aos efeitos prejudiciais da intervenção e voltados para o relato de resumos em jornais e conferências. ${ }^{14-19}$

Assim, reconhecida a relevância do enunciado CONSORT em promover o relato claro e completo das pesquisas em saúde e os esforços desse grupo em ampliar sua utilização através da extensão a diferentes estudos clínicos e a diversas línguas, este trabalho pretende abordar brevemente seus conceitos, auxiliando sua compreensão na língua portuguesa.

\section{Lista de Checagem (Checklist)}

As recomendações descritas abaixo foram fundamentadas segundo a versão revisada do enunciado CONSORT. ${ }^{4}$ Com o intuito de facilitar a interpretação e utilização das instruções, foi mantida a estruturação original em tópicos dos 22 itens da lista de checagem (Quadro 1) acompanhada de um exem$\mathrm{plo}^{20}$ de ECR especificando todas as etapas listadas ao final do texto (Quadro 2), cujo emprego auxilia o relato adequado de informações sobre o desenho, modo de condução e análise do estudo. Adicionalmente, as modificações propostas para atender às necessidades específicas de ECR não-farmacológicos ${ }^{18}$ foram convenientemente abordadas em alguns tópicos.

\section{Item 1- Título e Resumo}

Neste item é sugerido aos autores que declarem explicitamente o modo como os participantes foram distribuídos para os grupos experimentais e a inclusão desta informação no título é fortemente recomendada. Esta indicação surgiu da necessidade de identificar e indexar adequadamente ECR em bases eletrônicas, uma vez que muitos estudos são classificados de forma inapropriada. Para tanto, aconselha- 


\section{QUADRO 1- Lista de Checagem: Itens Necessários ao Relatar Estudos Clínicos Controlados Randomizados}

\begin{tabular}{|c|c|c|}
\hline Seção & Item & Recomendação CONSORT \\
\hline Título e Resumo & 1 & $\begin{array}{l}\text { Como os participantes foram alocados para } \\
\text { as intervenções ("alocação randomizada", "ran- } \\
\text { domizado", "randomicamente assinalados") }\end{array}$ \\
\hline \multicolumn{3}{|l|}{ Introdução } \\
\hline (Embasamento Científico) & 2 & $\begin{array}{l}\text { Contexto científico no qual a pesquisa se de- } \\
\text { senvolveu } \\
\text { Justificativa para realização do estudo }\end{array}$ \\
\hline \multicolumn{3}{|r|}{ 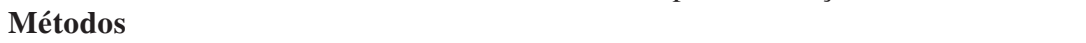 } \\
\hline Participantes & 3 & $\begin{array}{l}\text { Critérios de elegibilidade para os participan- } \\
\text { tes, os locais e ambientes onde os dados foram } \\
\text { coletados }\end{array}$ \\
\hline \multirow[t]{3}{*}{ Intervenções } & $4 \mathrm{~A}$ & $\begin{array}{l}\text { Descrições detalhadas sobre as intervenções } \\
\text { planejadas para cada grupo, como e quando } \\
\text { foram realmente administradas }\end{array}$ \\
\hline & 4B & \\
\hline & $4 \mathrm{C}$ & \\
\hline Objetivos & 5 & Especificar os objetivos e hipóteses do estudo \\
\hline Variáveis de Resposta & 6 & $\begin{array}{l}\text { Definir claramente as variáveis de resposta (pri- } \\
\text { márias e secundárias). Quando aplicável, des- } \\
\text { crever quaisquer métodos usados para melho- } \\
\text { rar a qualidade das mensurações (ex: treina- } \\
\text { mento dos avaliadores) }\end{array}$ \\
\hline Tamanho Amostral & 7 & $\begin{array}{l}\text { Como o tamanho da amostra foi determinado. } \\
\text { Quando aplicável, descrever e justificar quais- } \\
\text { quer análises intermediárias e regras para in- } \\
\text { terrupção do estudo }\end{array}$ \\
\hline $\begin{array}{l}\text { Geração da Sequência } \\
\text { de Alocação }\end{array}$ & 8 & $\begin{array}{l}\text { Método utilizado para gerar a sequência de } \\
\text { alocação randomizada, incluindo informações } \\
\text { sobre qualquer método de restrição (ex: por } \\
\text { blocos, estratificação) }\end{array}$ \\
\hline Mascaramento da Alocação & 9 & $\begin{array}{l}\text { Método utilizado para implementar a sequên- } \\
\text { cia de alocação randomizada (ex: frascos enu- } \\
\text { merados, tabela de números aleatórios), escla- } \\
\text { recendo se a sequência foi omitida até o mo- } \\
\text { mento da alocação dos participantes }\end{array}$ \\
\hline Implementação da Alocação & 10 & $\begin{array}{l}\text { Quem gerou a sequência de alocação, quem } \\
\text { recrutou os participantes e quem os assinalou } \\
\text { para os grupos }\end{array}$ \\
\hline Cegamento & $11 \mathrm{~A}$ & $\begin{array}{l}\text { Indicar se os participantes, os indivíduos que } \\
\text { administraram as intervenções e os que avalia- } \\
\text { ram os resultados eram cegos em relação ao } \\
\text { grupo de intervenção. }\end{array}$ \\
\hline
\end{tabular}

Quando aplicável, critérios de elegibilidade para os centros e para aqueles realizando as intervenções

Extensão para Estudos Não-Farmacológicos

Descrições detalhadas sobre ambos os tratamentos experimental e de comparação

Descrever os diferentes aspectos inerentes à intervenção.Quando aplicável, descrição dos procedimentos de adaptação das intervenções a cada participante

Detalhes sobre como as intervenções foram padronizadas

Detalhes sobre como foi avaliada ou alcançada a aderência dos profissionais de saúde ao protocolo

Quando aplicável, detalhar se os participantes foram aglomerados por centros e/ou profissionais de saúde e como isto foi realizado

Quando aplicável, como os profissionais de saúde foram alocados para cada grupo do estudo

Se aqueles administrando as co-internvenções eram cegos ou não em relação ao assinalamento dos grupos

Se realizado, descrever o método de cegamento e informações sobre o grau de similaridade entre as intervenções 
Métodos Estatísticos

\section{Resultados}

Fluxograma

Implementação da Novo

Intervenção Item

Recrutamento

Características Basais

Números Analisados

Resultados e Estimativas

Análises Adicionais

Efeitos Adversos

\section{Discussão \\ Interpretação}

Generalização

Evidências Vigentes
2 Métodos estatísticos usados pra comparar os grupos em relação às variáveis primárias de resposta; descrever quaisquer análises adicionais, como análises de subgrupo ou análises de ajuste.

13 Fluxo dos participantes durante cada estágio (recomenda-se a construção de um diagrama). Especificamente, descrever para cada grupo: o número de participantes que foram alocados randomicamente, os que receberam a intervenção planejada, os que completaram todo protocolo, os que foram incluídos nas análises estatísticas; descrever e justificar quaisquer desvios no protocolo pré-estabelecido

Datas definindo o período de recrutamento e acompanhamento

15 Características clínicas e demográficas da amostra, referentes ao período pré-intervenção

16 Número de participantes (denominador) em cada grupo incluídos em cada análise. Indicar se as análises foram por "intenção de tratar" (intention to treat analyses). Apresentar os resultados em números absolutos, se possível (ex: $5 / 15$, não $30 \%$ )

7 Descrever para cada resultado, primário e secundário, um sumário dos valores observados em cada grupo, o tamanho do efeito estimado acompanhado de sua precisão (ex: intervalo de confiança de $95 \%$ )

8 Indicar a realização de análises múltiplas, incluindo análises de subgrupos ou análises de ajuste. Informar se estas análises foram préestabelecidas ou exploratórias, ou seja, se foram planejadas ou sugeridas pelos achados.

19 Informar a ocorrência de eventos adversos ou efeitos colaterais

20 Interpretação dos resultados, levando em consideração as hipóteses do estudo, possíveis limitações e fontes potenciais de viéses, assim como, os riscos associados às análises múltiplas dos resultados

21 Generalização (validade externa) dos achados do estudo

22 Interpretação geral dos resultados sob o contexto das evidências vigentes
Quando aplicável, relatar se os participantes foram aglomerados segundo os centros ou profissionais de saúde e como isto foi realizado

O número de profissionais de saúde ou centros realizando a intervenção em cada grupo e o número de pacientes tratados por cada profissional de saúde ou em cada centro

Descrever o tratamento experimental e o de comparação da maneira como foram realmente implementados

Quando aplicável, descrição dos profissionais de saúde (volume de casos, qualificação, experiência etc.) e centros (nível de complexidade) em cada grupo

Adicionalmente, considerar a escolha da intervenção de comparação, o não cegamento ou sua realização parcial, o nível desigual de experiência dos profissionais de saúde ou centros em cada grupo

Generalização (validade externa) dos achados do estudo de acordo com a intervenção experimental e de comparação, pacientes, profissionais de saúde e centros envolvidos no estudo. 


\section{QUADRO 2- Lista de Checagem: Exemplo de Relato dos Itens Recomendado pelo Enunciado CONSORT}

\begin{tabular}{|c|c|c|}
\hline Seção & Item & Estudo Clínico Controlado Randomizado de Li et al. ${ }^{20}$ \\
\hline Título e Resumo & 1 & $\begin{array}{l}\text { Título: Efetividade do modelo de terapeuta primário para reabilitação da artrite reumatóide: } \\
\text { Um estudo clínico controlado e randomizado } \\
\text { Resumo: Comparar o modelo de terapeuta primário (MTP), fornecido por um único terapeuta } \\
\text { primário treinado em reumatologia, com o modelo de tratamento tradicional (MTT), fornecido } \\
\text { por um fisioterapeuta (FT) e/ou terapeuta ocupacional (TO) generalista, para tratar pacientes } \\
\text { com artrite reumatóide (AR). }\end{array}$ \\
\hline Introdução & 2 & $\begin{array}{l}\text { Profissionais de cuidado em saúde estão explorando novos modelos de serviço para atender às } \\
\text { demandas do tratamento de artrite. Para nosso conhecimento, nenhum estudo tem sido condu- } \\
\text { zido para avaliar a efetividade do MTP em contraposição ao MTT, que é o padrão da maioria } \\
\text { das facilidades canadenses para reabilitação da artrite. }\end{array}$ \\
\hline
\end{tabular}

\section{Métodos \\ Participantes}

Intervenções

Objetivos

Variáveis de Resposta

Tamanho Amostral
3 Paciente: Os candidatos elegíveis foram indivíduos que solicitaram FT e/ou TO e não tinham recebido tratamento de reabilitação para AR nos 2 últimos anos.

Terapeutas: Todos os terapeutas primários foram fisioterapeutas e terapeutas ocupacionais da Sociedade de Artrite que completaram o Programa de Treinamento em Avaliação de Poliartrites (34 FT, 14 TO). Os FT e TO tradicionais exerceram uma prática generalista em setor ambulatorial de hospitais (FT e TO), clínicas fundadas publicamente (principalmente FT) ou agências de cuidado domiciliar (FT e TO).

Recrutamento: Devido ao fato de o MTP ter sido utilizado somente pela Sociedade de Artrite e o MTT ser utilizado pela maioria das agências fundadas publicamente, nós conduzimos o ECR dentro do contexto destes 2 ambientes de serviço em Ontário. Houve 2 tipos de clínicas de fisioterapia fundadas publicamente: clínicas cujo proprietário é fisioterapeuta e clínicas cujo proprietário é medico e que contrata fisioterapeutas e outros profissionais da saúde.

4 A duração da intervenção foi de 6 semanas. Grupo MTP: Os terapeutas primários poderiam oferecer um ou uma combinação dos seguintes tratamentos: educação, incluindo o diagnóstico, controle da dor, conservação de energia e princípios de proteção articular e uso adequado de calçados; conselho quanto ao uso de modalidade física ou realização de exercícios específicos para artrite; conselho ou prescrição de equipamentos de assistência/ mobilidade (incluindo bengalas, andadores, cadeiras de roda e paciente); talas de membro superior/órteses para os pés; suporte psicossocial e de mediação entre os pacientes e administração da unidade de saúde.Grupo MTT: Todos os pacientes receberam educação no controle da dor. Em adição, fisioterapeutas puderam oferecer um ou uma combinação dos seguintes tratamentos: modalidades físicas para dor, conselho para exercício, conselho e prescrição de bengalas ou andadores. O tratamento da terapia ocupacional poderia incluir equipamentos de assistência (ex. aumento dos assentos de vaso sanitário), talas e órteses, e conselho e prescrição de protetores para mobilidade, incluindo bengala, andadores, cadeiras de roda e pacientes.

4A Não se aplica

4B Todos os terapeutas primários completaram o Programa de Treinamento em Avaliação de Poliatrites.

4C Terapeutas em ambos os grupos completaram um documento registrando os tratamentos oferecidos em cada sessão.

5 Comparar o modelo de terapeuta primário (MTP), fornecido por um único terapeuta primário treinado em reumatologia, com o modelo de tratamento tradicional (MTT), fornecido por um fisioterapeuta (FT) e/ou terapeuta ocupacional (TO) generalista, para tratar pacientes com artrite reumatóide (AR).

6 Medidas de resultado primárias: definidas como a proporção de respondentes clínicos que experimentaram melhora maior que $20 \%$ em 2 das seguintes medidas, obtidas pela comparação dos valores basais e de 6 meses: função física (medida pelo Health Assessment Questionnaire $[H A Q])$, dor (medida com a escala visual analógica [EVA]) e conhecimento da doença (medido com o Arthritis Community Research e Evaluation Unit RA Knowledge Questionnaire).

Medidas de resultado secundárias: diferença na mudança da pontuação para The Stanford Self Efficacy Scale, The RA disease activity index (RADAI) e The Coping Efficacy Scale.

7 Um total de 142 pacientes foi requerido pelo ECR, com base nas estimativas de um estudo piloto (diferença de respondentes clínicos entre grupos $=25 \%$, nível $\alpha=0.05$, poder $=80 \%$, taxa de atrito $=20 \%$ ). Nós estimamos um tamanho de amostra utilizando as medidas de resultado primárias. 
Geração da Sequência de Alocação

Métodos Estatísticos

\section{Resultados}

Fluxograma

Implementação

da Intervenção

Recrutamento

Características Basais

Números Analisados

Resultados e Estimativas

Análises Adicionais

Efeitos Adversos

\section{Discussão}

Interpretação

Generalização

Evidências Vigentes
8 Nós utilizamos o algoritmo de triagem da Sociedade de Artrite para assinalar o local de tratamento (ex. clínica ou domiciliar). Os pacientes foram estratificados pelo critério de classificação funcional do Colégio Americano de Reumatologia (CAR), previamente à randomização, já que a função física tem sido identificada como uma variável de prognóstico para a incapacidade. Nós assinalamos os pacientes elegíveis para o MTP ou MTT em uma taxa de 1:1, utilizando blocos de tamanho 6. Uma tabela de números randomizados gerada pelo computador foi utilizada para randomização.

9 Não mencionado

Não mencionado

11A/B Não houve cegamento dos pacientes e terapeutas. O artigo não deixa claro se a análise de dados foi cega.

Teste Qui-quadrado foi utilizado para analisar a associação entre o grupo de tratamento e a proporção de respondentes clínicos (medida de resultado primária).

Análises adicionais: Regressão logística foi utilizada para avaliar a associação do grupo de 12 tratamento com a medida de resultado primária através destes blocos de variáveis: variáveis demográficas do paciente (idade, sexo, nível de educação, rendimento médio da casa), variáveis relacionadas à doença (classe funcional do CAR, duração da doença). O nível de significância estatística foi de $P<0.05$ e foram calculados o Odds Ratio ajustado e o intervalo de confiança de $95 \%$. Medidas repetidas do teste ANOVA foram conduzidas para cada tratamento para explorar mudanças nas medidas de resultado com o transcorrer do tempo. Post-hoc LSD test foi também realizado.Teste T-Student foi utilizado para comparar a mudança nas pontuações para cada medida de resultado individual entre MTP e MTT em 2 momentos.

13 Os dados são extensos e por isso não foram representados nesta tabela. Por favor, veja maiores informações no artigo.

Novo Não mencionado ou não aplicável.

14 O recrutamento foi realizado entre novembro de 1999 e maio de 2002.

Idade, sexo, classe funcional do CAR, duração da doença, nível de educação, rendimento médio da casa, estado civil, estado de emprego, condições de moradia (sozinho, com família, casa de cuidados), medidas clínicas (dor, $H A Q$ e conhecimento).

16 Análises por tratamento: 111 pacientes foram incluídos nas análises de 144 pacientes randomizados.

17 Os dados são extensos e por isso não foram representados nesta tabela. Por favor, veja maiores informações no artigo.

18 Análises pré-especificadas: regressão logística. Análise exploratória: medidas repetidas do teste ANOVA e teste Post-hoc LSD.

19 Não mencionado

A discussão é extensa e por isso será apresentada parcialmente nesta tabela. Por favor, veja maiores informações no artigo.

20 O artigo apresenta muitas limitações do estudo que podem ser fontes potenciais de viés: Idealmente, uma análise por "intenção de tratar" deveria ser conduzida para avaliar a efetividade do tratamento. No entanto, isto não foi possível, de forma que os achados devem ser interpretados com cautela devido ao potencial viés de atrito.

21 Aspectos que devem ser considerados ao generalizar os resultados: Este estudo não incluiu clínicas de fisioterapia privadas para fornecer a terapia tradicional ao grupo MTT, sendo que em Ontário mais de 50\% das clínicas de fisioterapia são privadas. Além disso, o MTT no setor privado pode ser diferente do setor público. No entanto, como as clínicas privadas são disponíveis somente para alguns indivíduos, uma comparação envolvendo apenas estas clínicas não será generalizável para todos os pacientes que solicitam reabilitação para AR em Ontário.

22 Os resultados devem ser interpretados com cautela devido à alta taxa de atrito. No entanto, nossos achados concordam com estudos prévios nos quais os tratamentos foram fornecidos por um fisioterapeuta ou terapeuta ocupacional treinados em reumatologia. Outras pesquisas devem ser direcionadas a compreender a efetividade e o valor econômico dos profissionais de reabilitação com treinamento em reumatologia em diferentes modelos de saúde, tais como o modelo médico de terapia e a telemedicina. 
se o uso de expressões como "os pacientes foram randomicamente alocados" ou "o tratamento foi randomizado" entre outras.

O resumo deve ser suficientemente detalhado e transparente, uma vez que é o responsável por motivar a leitura do texto completo e, não raro, representa a única fonte de acesso a informações que possivelmente nortearão as decisões clínicas baseadas em evidências. Os aspectos centrais do resumo, que devem ser mencionados a fim de permitir a avaliação da validade e aplicabilidade do estudo, podem ser consultados em Hopewell et al. ${ }^{19}$ Resumos estruturados em subtópicos com detalhes referentes ao desenho, modo de condução, análise e conclusões do estudo garantem uma maior qualidade e facilidade na busca de informações quando comparados aos resumos tradicionalmente descritos. Em relação aos estudos clínicos não-farmacológicos, recomenda-se a descrição de dados sobre os centros de recrutamento dos pacientes, o número e nível de experiência dos profissionais de saúde envolvidos, os diferentes grupos de comparação e quais indivíduos foram mantidos cegos durante o estudo.

\section{ITEM 2- Introdução}

A introdução geralmente expõe o contexto científico no qual a pesquisa está sendo desenvolvida e deve conter a justificativa para realização do referido estudo, já que a exposição desnecessária de seres vivos aos riscos inerentes à investigação científica é eticamente desaconselhável pela Declaração de Helsinki (1997). ${ }^{21}$ Além disso, é preciso indicar os prováveis benefícios da intervenção, bem como as explicações plausíveis para suas ações, principalmente quando existe pouca ou ainda nenhuma experiência prévia em relação à intervenção a ser investigada. A inclusão de uma revisão sistemática de estudos clínicos similares ou a notificação da ausência dos mesmos é sugerida, sendo também apropriada a descrição dos objetivos.

\section{Métodos}

\section{ITEM 3- Participantes}

Este item orienta quanto aos participantes da pesquisa, que são definidos pelos critérios de inclusão e pelo local e forma de recrutamento.

\section{ITEm 3.a) Os critérios de inclusão}

Precisam ser descritos claramente, não sendo necessária a distinção com os critérios de exclusão.
Nos estudos não-farmacológicos, os critérios de inclusão se estendem aos centros e aos responsáveis por realizar as intervenções, pois o nível de complexidade dos centros de coleta e o nível de experiência dos profissionais de saúde podem afetar o efeito estimado da intervenção em análise. Para tanto, o artigo deve relatar o número e o tipo de centros e de profissionais de saúde envolvidos no estudo.

\section{ITEM 3.b) Local de coleta e recrutamento}

Particularmente importante é o relato de quaisquer fatores que comprometam a validade externa do estudo, como o local de coleta e recrutamento, se hospitais, clínicas ou comunidade e a forma de recrutamento, que pode ser caracterizada por anúncios ou comparecimento voluntário, por encaminhamentos, dentre outras.

\section{ITEM 4- Intervenções}

Este item refere-se à descrição das intervenções planejadas para cada grupo e como e quando elas foram realmente administradas. $\mathrm{O}$ grupo controle pode se apresentar em diferentes formas através de uma intervenção usual, uma intervenção ativa, um placebo ou ainda uma lista de espera. A informação clara e completa de todos os procedimentos envolvidos na intervenção é essencial para facilitar a comparação do estudo, a reprodutibilidade e sua inclusão em revisões sistemáticas. Em estudos não-farmacológicos, como os de intervenção fisioterapêutica, é necessário descrever o conteúdo, o número e duração das sessões, duração da intervenção, os instrumentos utilizados para transmitir a informação, se a intervenção foi monitorada e se foi individual ou em grupo. Neste sentido, também é importante descrever procedimentos para padronizar a intervenção oferecida, possibilitando o acesso a esse material através de um apêndice ou de um link on-line. Outro aspecto relevante é o nível de aderência dos profissionais envolvidos ao protocolo proposto, pois isto pode influenciar a reprodutibilidade e aplicabilidade futura dos achados.

\section{Item 5- Objetivo e hipótese}

Segundo recomendado por este item, os autores devem especificar os objetivos e hipóteses do estudo. Os objetivos representam as questões que o estudo se propôs a responder ou investigar, enquanto que as hipóteses se referem aos resultados esperados, ou ainda às respostas provisórias, que ao serem confirmadas auxiliam os objetivos. ${ }^{4}$ 


\section{ITEM 6- Variáveis de resposta}

Este item diz respeito às variáveis de resposta, distinguidas em primárias e secundárias. A variável de resposta primária é a variável de maior relevância para o estudo, sendo pré-especificada e utilizada para o cálculo do tamanho da amostra. As demais variáveis de interesse do estudo representam as variáveis secundárias, que não necessariamente são planejadas antes do início do estudo. A mensuração das variáveis deve priorizar a utilização de escalas e instrumentos validados, o que garante a qualidade das medidas e permite a comparação com estudos similares. A descrição das mensurações deve ser feita em relação ao início, periodicidade e término e para qualquer método utilizado para melhorar sua qualidade, tais como a realização da mensuração por mais de um avaliador, o treinamento dos avaliadores e a manutenção dos avaliadores "cegos" quanto aos grupos de intervenção.

\section{Item 7- Tamanho amostral e análises inter- mediárias}

\section{ITEm 7.a) Tamanho da amostra}

A determinação do tamanho da amostra requer um planejamento cuidadoso, sendo realizada antes de iniciar o estudo. A amostra precisa ser grande o suficiente para possibilitar uma alta probabilidade de detectar diferenças estatisticamente e clinicamente significativas, se estas existirem. Alguns estudos são marcados pela diferença entre o tamanho da amostra planejado e real ao término da pesquisa devido à interrupção precoce, possivelmente motivada por razões éticas, replanejamento do tamanho amostral e dificuldade de recrutamento. Nesses casos, geralmente se verifica a utilização de análises intermediárias.

\section{ITEm 7.b) Análises intermediárias}

As análises intermediárias são geralmente utilizadas para decidir se o recrutamento deve continuar, aspecto este que precisa ser mencionado no artigo quanto à quantidade e tipo de análises realizadas e se estas foram planejadas previamente ao início do estudo ou algum tempo depois. Em relação aos estudos não-farmacológicos, é importante relatar se as análises intermediárias foram feitas por aglomerado, sendo cada aglomerado representado pelos indivíduos que receberam a intervenção de um mesmo profissional de saúde. Este tipo de análise deve ser considerado, uma vez que os indivíduos não são capazes de gerar observações independentes por terem recebido a intervenção de um mesmo pesquisador/profissional de saúde.

\section{ITENS 8 e 9- Referentes à alocação}

A qualidade dos ECR está fortemente associada ao processo de randomização, que pode ser entendido pelas etapas de gerar uma sequência de alocação randomizada (Item 8) e de mascarar esta sequência (Item 9) de forma a não permitir que os investigadores envolvidos com o recrutamento dos indivíduos a conheçam previamente. Assim, os autores devem especificar o método utilizado para gerar esta sequência de alocação randomizada (Item 8.a), se randomizado ou quase-randomizado. A randomização verdadeira ocorre quando o indivíduo é assinalado para um grupo com base no acaso, ou seja, tem igual chance de ser distribuído/alocado para qualquer um dos grupos de comparação. Para tanto, sugere-se a utilização de uma tabela de números aleatórios, uma sequência de números randomizados gerada pelo computador ou o ato de jogar uma moeda. Nos métodos quase-randomizados, a chance do indivíduo ser assinalado para um determinado grupo já está definida antes mesmo dele entrar em contato com o investigador. Este tipo de alocação determinística, em vez de pelo acaso, ocorre sempre que os indivíduos são assinalados para os grupos de acordo com seu número de registro em uma instituição de saúde, número de carteira de identidade, data de nascimento ou ordem de chegada, se par ou ímpar.

A randomização simples pode também ser substituída pela randomização restrita (Item 8.b) através da utilização de métodos que visam equilibrar os grupos quanto ao número de indivíduos (randomização em bloco) ou quanto a algumas características da linha de base, cuja diferença entre os grupos poderia enfraquecer a credibilidade do estudo (randomização por estratos). Para os estudos não-farmacológicos, o processo de randomização deve ser relatado também para os profissionais de saúde.

O mascaramento da sequência de alocação randomizada é de considerável importância, pois sua não realização pode invalidar o processo anterior de geração da sequência. Consiste em prevenir o conhecimento prévio da intervenção a ser assinalada, evitando que aqueles envolvidos com o recrutamento dos indivíduos sejam influenciados por este conhecimento. Tal processo, sempre possível de ser realizado até o momento em que o indivíduo é assinalado para um dos grupos de intervenção, previne o viés de seleção e precisa ser claramente distinguido do processo de cegamento que se inicia somente após o término da seleção e nem sempre é possível. 
Os autores devem relatar os procedimentos utilizados para mascarar a sequência, sendo desejável o envolvimento externo de indivíduos não relacionados ao estudo e, quando não viável, a utilização de envelopes opacos ou frascos, ambos selados e numerados de acordo com a sequência aleatória previamente gerada. É fundamental assegurar que os envelopes ou frascos sejam abertos somente após serem registrados com o nome do indivíduo e outros detalhes pertinentes.

\section{ITEM 10- Implementação}

O planejamento do estudo deve ser cauteloso quanto a definir claramente aqueles que geraram a sequência, que registraram os indivíduos e que os assinalaram para os determinados grupos. O estudo pode ser diferenciado em dois procedimentos definidos por 1) gerar e mascarar a sequência de alocação randomizada e 2) procedimento de implementação dos assinalamentos para cada grupo. Faz-se necessário separar completamente os indivíduos envolvidos em cada procedimento, uma vez que aquele responsável por assinalar os indivíduos pode determinar o grupo para o qual o indivíduo será alocado, se esteve envolvido com o processo de gerar a sequência de alocação. Assim, um indivíduo pode desempenhar mais de uma função dentro do procedimento de gerar e mascarar a sequência, mas não pode estar envolvido com a implementação e vice-versa.

\section{ITEM 11- Cegamento}

O cegamento consiste em manter a intervenção assinalada desconhecida pelos participantes, profissionais de saúde que administram a intervenção e aqueles coletando os dados clínicos e analisando os resultados. Considerando as diversas possibilidades de cegamento, os autores devem relatar claramente para quem o estudo foi cego, ao invés de utilizar termos como "duplo cego", sem maiores esclarecimentos. A função desempenhada pelo processo de cegamento é específica a cada indivíduo para o qual é realizado. No caso de cegamento dos pacientes, tenta-se prevenir o viés de execução resultante de efeitos não associados com a intervenção recebida, sendo este efeito melhor evidenciado com a utilização de grupo controle placebo. $\mathrm{O}$ efeito placebo pode ser traduzido em expectativas positivas ou ansiedade do paciente em relação a um tratamento novo, bem como a baixas expectativas ou tranquilidade do paciente submetido a um tratamento usual. O cegamento dos profissionais de saúde que administram a intervenção também previne o viés de execução, enquanto que o cegamento daqueles que coletam as variáveis de resposta e analisam os resultados previne o viés de detecção, evitando-se que disponham de atitudes e estímulos diferenciados aos pacientes por reconhecer para qual intervenção ele foi assinalado ou que selecionem os métodos estatísticos baseados neste conhecimento prévio.

Alguns estudos não-farmacológicos apresentam limitações para o cegamento, uma vez que as intervenções envolvem procedimentos conhecidos tanto por aquele que as administram como pelo paciente. Além disso, sinais e sintomas não previstos podem evidenciar ao paciente e ao profissional da saúde a intervenção recebida e administrada, comprometendo o cegamento previamente planejado. A realização do cegamento daqueles coletando as variáveis de resposta e analisando os resultados é particularmente importante nestes casos marcados pela impossibilidade ou falhas de cegamento, sendo também recomendada em estudos cujas variáveis de resposta são subjetivas, como no caso do relato de dor.

Os desafios encontrados pelos estudos não-farmacológicos para adotar estratégias de cegamento requer aos autores relatarem detalhadamente o método utilizado e as similaridades entre as intervenções, contribuindo com a exploração de formas diferentes de implementar o cegamento.

\section{ITEM 12- Métodos estatísticos}

Os autores devem especificar os procedimentos estatísticos utilizados durante todas as etapas de realização do estudo e se estes foram planejados previamente ou sugeridos pelos achados. Geralmente, as análises fornecem estimativas quanto à eficácia da intervenção proposta ao comparar as variáveis primárias de resposta observadas em cada grupo. Aconselha-se, preferencialmente, a descrição dos intervalos de confiança do referido efeito estimado da intervenção em vez da utilização do $P$ valor, que deve ser mencionado em valores exatos, se empregado. As análises adicionais, distinguidas em análises subgrupo e análises de ajuste, devem ser realizadas com cautela e se possível pré-especificadas, pois muitas vezes resultam em inferências equivocadas. As análises de subgrupo visam identificar possíveis diferenças na eficácia do tratamento proposto entre subgrupos complementares, como por exemplo, ao estimar se os resultados diferem entre os indivíduos de um mesmo grupo de tratamento segundo características específi- 
cas, como por exemplo, sexo e faixa etária. As análises de ajuste, também desencorajadas pelo enunciado CONSORT, representam quaisquer procedimentos utilizados para homogeneizar as amostras em relação a alguns aspectos da linha de base e, se necessárias, não devem ser determinadas pela diferença de $P$ valor, mas por serem reconhecidas previamente como importantes fatores prognósticos.

\section{Resultados}

\section{ITEM 13- Fluxograma dos participantes}

A interpretação dos resultados de ECR é influenciada pela maneira como são descritas informações sobre a distribuição dos participantes durante cada etapa do estudo. Detalhes relativos ao recrutamento, alocação e seguimento dos indivíduos devem ser especificados para ambos os grupos. Os autores são orientados a relatar: o número de participantes entrevistados e consecutivamente selecionados para o estudo; o número de indivíduos alocados em cada grupo e quantos destes receberam de fato a intervenção proposta conforme planejada; o número de indivíduos desistentes ou dispensados durante o seguimento, assim como o número de indivíduos incluídos nas análises finais. Em estudos não-farmacológicos é necessário descrever o número de centros e profissionais envolvidos. A construção de um fluxograma é fortemente recomendada (Figura 1).

Adicionalmente, os autores devem informar e justificar quaisquer eventuais desvios no protocolo previamente proposto, tais como modificações nos métodos de análise dos dados, alterações nas avaliações

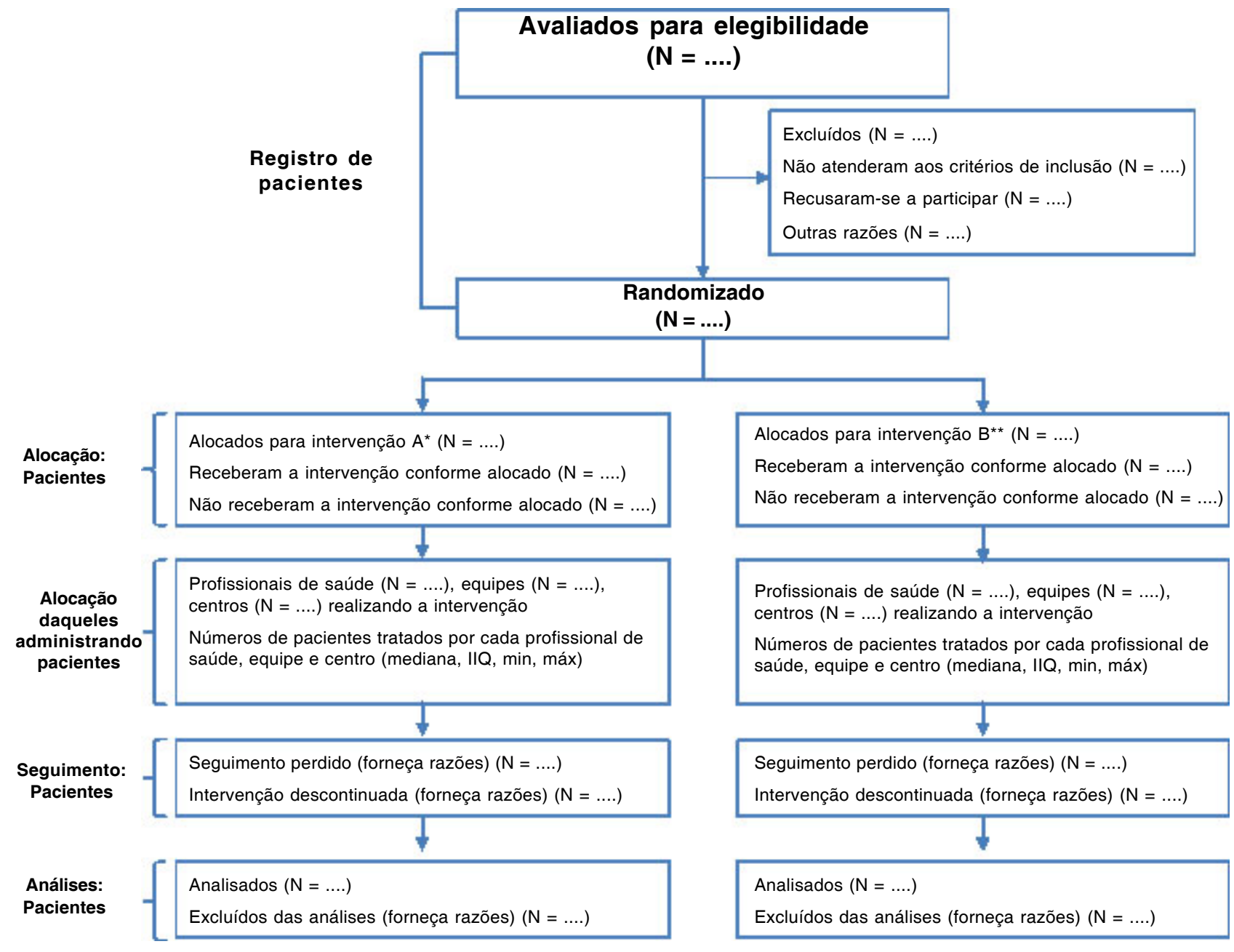

Figura 1: Diagrama representando o fluxo dos participantes em cada etapa do estudo. ${ }^{*} A=$ Intervenção investigada; ${ }^{* *} B=$ Intervenção controle. 
ou ainda nas intervenções após a alocação dos indivíduos. O uso do termo "desvio de protocolo" não é suficiente para justificar a exclusão de participantes após a randomização.

\section{ITEM 14- Recrutamento e acompanhamento}

Os autores devem descrever claramente o período em que foi realizado o recrutamento, como também informar durante quanto tempo os participantes foram acompanhados e se houve encerramento do estudo antes do previsto. Especificações quanto à taxa de recrutamento (número de participantes recrutados em determinado período de tempo), e se este ocorreu conforme planejado podem ser úteis a outros pesquisadores.

\section{ITEM 15- Características clínicas e demográ- ficas da amostra}

Neste item é recomendada a descrição das características clínicas e demográficas da amostra referentes ao período anterior à intervenção, facilitando assim o julgamento, por parte do leitor, quanto às particularidades dos resultados, sua relevância e aplicabilidade clínica em indivíduos com características semelhantes às da amostra submetida ao tratamento. A comparação destas características no período anterior à intervenção permite avaliar similaridades entre os grupos. Ressalta-se, porém, que a alocação randomizada dos participantes visa garantir que possíveis diferenças entre os grupos sejam advindas do acaso e não consequentes a viéses de seleção.

De maneira prática, os dados podem ser apresentados em tabelas. Sugere-se o uso de medidas de tendência central e suas respectivas medidas de dispersão na descrição de variáveis quantitativas contínuas. Já em relação às variáveis qualitativas ou quantitativas discretas, como por exemplo, níveis de severidade da doença ou número de partos, respectivamente, devem ser apresentadas segundo a frequência ou proporção observadas em cada grupo.

\section{ITEM 16- Número de participantes incluídos nas análises}

Como já anteriomente descrito no item 15, os autores devem relatar o número de participantes que foram incluídos em cada grupo em cada análise do estudo. Faz-se necessário indicar se as análises foram feitas por "intenção de tratar" ( "intention to treat analyses"), ou seja, todos os indivíduos foram analisados conforme alocados. Para tanto, os resultados de cada grupo devem ser apresentados em números absolutos e não em números fracionados (i.e., $5 / 15$ e não 30\%), facilitando a identificação de falhas relativas a não inclusão de participantes em todas as análises, uma vez que a exclusão destes, por desistência ou não aderência ao protocolo, pode acarretar em conclusões errôneas quanto ao efeito estimado da intervenção.

\section{ITEM 17- Variáveis de resposta, efeito esti- mado e intervalo de confiança}

Os autores devem formular um sumário representando todas as variáveis de interesse analisadas pelo estudo e utilizadas para o cálculo do tamanho do efeito estimado do tratamento. $\mathrm{O}$ tamanho do efeito estimado deve ser descrito para ambos os grupos em conjunto, com os respectivos intervalos de confiança. $\mathrm{O}$ tamanho do efeito estimado segundo variáveis binárias (p. ex., presença ou não de determinada característica) pode ser representado por meio de valores de risco relativos, odds ratio, entre outros, enquanto que para variáveis contínuas, o tamanho do efeito pode ser demonstrado, em conjunto com os intervalos de confiança, por meio da diferença entre as médias obtidas através da comparação entre os grupos. Estes valores são mais facilmente interpretados se forem disponibilizados em tabelas ao invés de descritos ao longo do texto. Orienta-se também a descrição de todas as variáveis de reposta conforme definidas previamente às intervenções e não somente as variáveis que obtiveram diferenças estatisticamente significativas.

\section{ITEM 18- Análises adicionais}

Apesar de análises planejadas previamente fornecerem resultados mais confiáveis, análises exploratórias múltiplas realizadas posteriormente à intervenção e sugeridas pelos dados são comumente utilizadas nos estudos, mesmo que ofereçam um alto risco de superestimar os resultados. ${ }^{4}$ Por este motivo, os autores são orientados a especificar se as análises utilizadas no estudo foram planejadas ou sugeridas pelos achados. Recomenda-se ainda, conforme descrito no item 12, a descrição de análises de ajuste, sendo aconselhável apresentar ambos os resultados, ou seja, com e sem tais análises.

\section{ITEM 19- Eventos adversos e efeitos colaterais}

Os autores devem informar a ocorrência de desfechos indesejáveis durante a realização da investigação, como eventos adversos ou efeitos colaterais. 
Apesar de não-intencionais, a existência de tais desfechos prejudica a aplicabilidade futura dos achados clínicos do estudo, tornando necessário informar os leitores sobre os possíveis riscos e benefícios associados à intervenção proposta. Os eventos adversos são consequências, em muitos casos, da condição tratada, enquanto que os efeitos colaterais são decorrentes da intervenção proposta. Os autores devem indicar o número de indivíduos afetados, a frequência e a natureza dos desfechos mais graves, o método utilizado na mensuração dos mesmos, justificando eventuais descontinuidades no tratamento.

\section{Discussão}

\section{ITEM 20- Interpretação}

Este item sugere que os autores elaborem uma discussão cuidadosa dos resultados observados, interpretando-os segundo a(s) hipótese(s) do estudo. Basicamente, a discussão do trabalho deve conter: 1) uma sinopse dos resultados principais; 2) considerações sobre os possíveis mecanismos responsáveis pelos desfechos; 3) argumentação crítica dos achados de acordo com a literatura disponível, sendo desejável a inclusão de uma revisão sistemática, sempre que disponível; 4) informações referentes aos eventuais riscos e limitações do estudo, assim como, sobre os procedimentos utilizados para minimizar estes problemas; 5) um sumário final com as conclusões sobre as implicações clínicas e científicas relevantes. Em estudos não-farmacológicos, os autores devem discutir as implicações terapêuticas da intervenção controle escolhida (placebo, tratamento tradicional, lista de espera) em contraste com a intervenção investigada, já que em muitos casos, os procedimentos controles são questionáveis por subestimarem a eficácia da intervenção proposta. O nível de experiência, e se esta diferiu entre os investigadores envolvidos com a intervenção, é outro fator relevante que deve ser abordado pelos autores. Adicionalmente, recomenda-se a discussão de aspectos referentes às limitações e viabilidade do cegamento.

\section{ITEM 21- Generalização}

Complementares ao item 20 devem ser considerados alguns aspectos relativos à validade externa do estudo, como as características dos participantes, do local e da equipe, do regime de tratamento, entre outros relevantes. Deste modo, os autores devem indagar e sugerir se os achados são aplicáveis, por exemplo, a outros indivíduos com idade, sexo ou comorbidades diferentes, ou ainda, se resultados similares podem ser obtidos em outros serviços com níveis de complexidade diversos, isto é, serviços públicos ou privados; níveis de complexidade primária, secundária ou terciária. A discussão de tais aspectos permite avaliar a extensão na qual os resultados do estudo são generalizáveis a outras circunstâncias, auxiliando o leitor no processo de tomada de decisão baseada em evidências científicas.

\section{ITEM 22- Interpretação geral segundo evidên- cias}

Finalmente, ao concluir o trabalho, os autores devem apresentar uma interpretação global dos resultados sob o contexto da evidência científica vigente. Recomenda-se, portanto, confrontar os resultados com dados da literatura existente sobre o tema, sendo desejável a inclusão de uma revisão sistemática de ECRs similares. A existência de poucos ou nenhum estudo semelhante prévio deve ser mencionada. Além de promover suporte aos achados, tais discussões colaboram com a divulgação de outros estudos relevantes, como também dos procedimentos mais usados e efetivos sobre o tema em investigação.

\section{Considerações Finais}

Conforme exemplificado pelas recomendações acima, o enunciado CONSORT tem como objetivo auxiliar o relato completo e transparente de informações de ECR de modo a refletir na melhora da qualidade das informações divulgadas na literatura de saúde e assim, viabilizar a prática das idéias preconizadas pela prática baseada em evidências. $\mathrm{O}$ presente trabalho pretendeu, portanto, facilitar a compreensão de tais instruções, assim como divulgá-las em Língua Portuguesa do Brasil, ao abordar brevemente os principais aspectos contidos na versão revisada do enunciado CONSORT. ${ }^{4}$ Sugerimos ainda, que algumas destas recomendações não sejam conferidas somente para o relato adequado de informações relevantes ao término das investigações científicas, mas que sejam também empregadas já na fase de planejamento das mesmas, prevenindo surpresas desagradáveis ou desfechos inapropriados e conseguinte limitação das conclusões realizadas. 


\begin{abstract}
Clinical strategies and decision making in health care should be supported by the best available scientific evidence not only on intuition neither on previous clinical experience. Randomized controlled trials (RCT) are considered the gold standard for the assessment of interventions efficacy but inadequate report difficulties their interpretation and identification of eventual bias. A group of scientists and editors developed the CONSORT Statement to improve the quality of reporting of RCTs. The CONSORT statement comprises a checklist of essential items that should be included in reports of RCTs and a diagram for documenting the flow of participants through a trial. Nowadays, the CONSORT are translated to several languages and it has been modified to attend different study designs. The present document intended to improve the comprehension of CONSORT recommendations and to spread its use on Portuguese language through a brief discussion of main aspects from the revised version of CONSORT statement.
\end{abstract}

Keywords: CONSORT. Controlled Clinical Trials as Topic. Randomized Controlled Trial. EvidenceBased Medicine.

\section{Referências}

1. Sackett DL, Rosenberg W, Haynes RB, Richardson S. Evidence-based medicine: what it is and what it isn't. BMJ 1996; 312: 71-2.

2. Atallah AN, Castro AA. Evidências para melhores decisões clínicas. Disponível em: http://www.centrocochranedobrasil. org.br/artigos/bestevidence.htm Acesso em 13 dez 2008.

3. Ho PM, Peterson PN, Masoudi FA. Evaluating the evidence: is there a rigid hierarchy? Circulation. 2008; 118:1675-84.

4. Altman DG, Schulz KF, Moher D, Egger M, Davidoff F, Elbourne $\mathrm{D}$ et al. The revised CONSORT statement for reporting randomized trials: explanation and elaboration. Ann. intern. med. 2001; 134: 663-94.

5. Schriger DL, Arora S, Altman DG. The content of medical journals instruction for authors. Ann. intern. med. 2006; 48 743-9.

6. International Committee of Medical Journal Editors (ICMJE): Uniform Requirements for Manuscripts Submitted to Biomedical Journals. Disponível em: http://www.icmje.org Acesso em 13 dez 2008.

7. Moher D, Simera I, Schulz KF, Hoey J, Altman DG. Helping editors, peer reviewers and authors improve the clarity, completeness and transparency of reporting health research. BMC Medicine 2008; 6:13.

8. Bossuyt PM, Reitsma JB, Bruns DE, Gatsonis CA, Glasziou PP, Irwig LM et al. The STARD Statement for Reporting Studies of Diagnostic Accuracy: Explanation and Elaboration. Clin. chem. 2003; 49: 7-18.

9. Moher D, Cook DJ, Eastwood S, Olkin I, Rennie D, Stroup DF Improving the quality of reports of meta-analyses of randomised controlled trials: the QUOROM statement: quality of reporting of meta-analyses. Lancet .1999; 354:1896900.

10. Stroup DF, Berlin JA, Morton SC, Olkin I, Williamson GD, Rennie $D$ et al. Meta-analysis of observational studies in epidemiology: a proposal for reporting: Meta-analysis Of Observational Studies in Epidemiology (MOOSE) group. JAMA. 2000; 283: 2008-12.

11. Altman DG. Better reporting of randomized controlled trials: the CONSORT statement [Editorial]. BMJ 1996; 313: 570-1.
12. Devereaux PJ, Manns BJ, Ghali WA, Quan H, Guyatt GH. The reporting of methodological factors in randomized controlled trials and the association with a journal policy to promote adherence to the Consolidated Standards of Reporting Trials (CONSORT) checklist. Control. clin. trials. 2002; 23: 3808.

13. Plint AC, Moher D, Schulz K, Altman DG, Morrison A. Does the CONSORT checklist improve the quality of reports of randomized controlled trials? A systematic review. Med. j. Aust. 2006; 185: 263-7.

14. Campbell MK, Elbourne DR, Altman DG, CONSORT Group. CONSORT statement: extension to cluster randomized trials. BMJ 2004; 328: 702-8.

15. Piaggio G, Elbourne DR, Altman DG, Pocock SJ, Evans SJ, CONSORT Group. Reporting of noninferiority and equivalence randomized trials: an extension of the CONSORT statement. JAMA. 2006; 295:1152-60.

16. Gagnier JJ, Boon H, Rochon P, Moher D, Barnes J, Bombardier C, CONSORT Group. Reporting randomized, controlled trials of herbal interventions: an elaborated CONSORT statement. Ann. intern. med. 2006; 144: 364-7.

17. Ioannidis JP, Evans SJ, Gotzsche PC, O'Neill RT, Altman DG Schulz K, CONSORT Group. Better reporting of harms in randomized trials: an extension of the CONSORT statement. Ann. intern. med. 2004; 141: 781-8.

18. Boutron I, Moher D, Altman DG, Schulz KF, Ravaud P. Extending the CONSORT statement to randomized trials of nonpharmacologic treatment: explanation and elaboration. Ann. intern. med. 2008; 295-309.

19. Hopewell S, Clarke M, Moher D, Wager E, Middleton P, Altman DG, Schulz KF, The CONSORT Group. CONSORT for reporting randomised trials in journal and conference abstracts. Lancet. 2008; 371: 281-3.

20. Li CL, Davis AM, Lineker SC, Coyte PC, Bombardier C. Effectiveness of the primary therapist model for rheumatoid arthritis rehabilitation: a randomized controlled trial. Arthritis rheum. 2006; 55:42-52.

21.World Medical Association Declaration of Helsinki. Recommendations guiding physicians in biomedical research involving human subjects. JAMA. 1997; 277: 925-6. 